Revue

d'ethnoécologie
Revue d'ethnoécologie

$9 \mid 2016$

Varia + dossier "Cartographie participative" (1)

\title{
« Mettre en carte » le territoire
}

Les enjeux de la cartographie participative aux Suds

\section{Pascale de Robert et Stéphanie Duvail}

\section{(2) OpenEdition}

\section{Journals}

Édition électronique

URL : http://journals.openedition.org/ethnoecologie/2739

DOI : $10.4000 /$ ethnoecologie.2739

ISSN : 2267-2419

Éditeur

Laboratoire Eco-anthropologie et Ethnobiologie

Référence électronique

Pascale de Robert et Stéphanie Duvail, « « Mettre en carte » le territoire », Revue d'ethnoécologie [En ligne], 9 | 2016, mis en ligne le 01 juillet 2016, consulté le 04 mai 2019. URL : http://

journals.openedition.org/ethnoecologie/2739; DOI : 10.4000/ethnoecologie.2739

Ce document a été généré automatiquement le 4 mai 2019.

\section{c)}

Revue d'ethnoécologie est mis à disposition selon les termes de la licence Creative Commons Attribution - Pas d'Utilisation Commerciale - Pas de Modification 4.0 International. 


\title{
« Mettre en carte » le territoire
}

\author{
Les enjeux de la cartographie participative aux Suds
}

\author{
Pascale de Robert et Stéphanie Duvail
}

1 Mettre en carte un territoire habité, une terre d'où sont prélevées des ressources, un espace parcouru qui est aussi un lieu de mémoire, de savoirs, voire de promesses, n'est jamais un exercice anodin. La pratique cartographique implique la collaboration de différents acteurs du territoire et d'experts aux profils variés, avec autant de perceptions différenciées de ces espaces. Dès lors, le choix des éléments ou phénomènes à inclure sur la carte, à mettre en valeur, à rendre visible et, en négatif, le choix d'exclure d'autres éléments du paysage, demande un travail de concertation qui disparait bien souvent du document final. Pourtant, aussi complète et fournie soit-elle, une carte n'est jamais qu'une représentation graphique en deux ou trois dimensions d'une réalité plus complexe, un point de vue donné sur le monde : une carte est toujours subjective.

2 Si ces évidences méritent d'être rappelées c'est parce que les cartes sont très souvent sollicitées lors de situations conflictuelles à propos d'un espace ou au moment de prendre des décisions d'aménagement. Parce qu'elles sont utilisées comme médiateurs de relations de pouvoir à propos de l'espace et parce qu'elles reconfigurent ces relations de pouvoir, les cartes sont avant tout des objets politiques, qu'il convient d'étudier en tant que tels.

3 Alors que la cartographie traditionnelle est longtemps restée l'apanage des Etats, on assiste depuis les années 1970 à un foisonnement d'expériences de cartographie dite " participative ». Ce mouvement, amorcé avec les premières expériences de cartographie engagée de Paolo Freire s'est affirmé depuis les années 2000 avec la multiplication des expériences de type MARP (Méthode Accélérée de Recherche Participative) au sein des Organismes Non Gouvernementaux (ONG) et des services de l'Etat, et la démocratisation des outils de Systèmes d'Information Géographique (SIG) et de télédétection. Ces expériences sont très diverses dans leurs approches et méthodes mais revendiquent toutes une participation active des habitants d'un territoire à la production de sa cartographie. 
4 La cartographie participative interroge les chercheurs en sciences sociales à plusieurs titres, et en tant qu'utilisateurs en premier lieu: demander aux populations étudiées de produire une carte de leur territoire est un exercice utile aux chercheurs en sciences sociales, en complément des outils classiques de l'anthropologie sociale ou de la géographie pour une meilleure spatialisation des phénomènes sociaux et une meilleure représentation des savoirs locaux liés à l'espace. La cartographie revisitée par ceux qui habitent les lieux dessinés sur le papier - ou « ethno-cartographie » comme préfèrent la nommer plusieurs auteurs de ce dossier - est une méthode riche, productrice de nouvelles informations, mais elle n'est pas sans soulever de nouvelles questions méthodologiques. Par ailleurs, parce que les cartes sont subjectives, susceptibles de servir des stratégies politiques et qu'on assiste à une inflation de leur utilisation, elles sont également pour les chercheurs en sciences sociales de passionnants objets d'étude, à analyser dans le contexte de leur élaboration: c'est la cartographie ou le processus de « mise en carte » d'un territoire, plus que la carte elle-même en tant que produit qui nous intéresse dans ce dossier.

Dans ces exemples choisis au Sud, et pour cette première partie du dossier ${ }^{1}$, en Amérique Latine et en Afrique, la collaboration des chercheurs - impliqués depuis longtemps par leurs recherches respectives auprès des villages concernés - a été formalisée dès le début du processus d'élaboration de la carte, mais dans des contextes et avec des objectifs différents. A partir d'études de cas, les articles de ce dossier présentent les processus de production des cartes par ces nouveaux cartographes que sont les habitants, autochtones ou collectifs locaux, accompagnés ou pas dans leur entreprise par des ONG et services publics. Les auteurs se sont attachés à présenter les étapes et les implications d'expériences de cartographie menées par des communautés locales dont les territoires, l'histoire, revendications ou savoirs restaient « invisibles » sur les cartes préexistantes. Ils proposent d'analyser le contexte géopolitique, les motivations et les processus qui mènent dans chaque cas à la cartographie. Ces exemples variés orientent notre réflexion dans plusieurs directions.

\section{La cartographie est révélatrice des tensions sur le territoire}

6 La cartographie participative au Sud, est souvent pratiquée dans des situations que l'on peut qualifier de post-coloniales, ou dans des contextes où le foncier et les ressources naturelles sont disputés.

Dans les cas d'étude présentés dans la première partie de ce dossier, les cartographies sont réalisées par des populations dont les territoires sont soit officiellement reconnus par l'Etat (Brésil, Roselis Mazurek), soit en passe d'être légalement homologués par l'État (Venezuela, Hortencia Caballero, Stanford Zent et al.), soit encore susceptibles d'être valorisés comme hauts lieux historiques (Guyane, Marie Fleury et al.) ou espace de conservation de la biodiversité (Sénegal et Mauritanie, Marie-Christine Cormier-Salem et al.). Dans ces différents exemples, la cartographie est révélatrice de ces tensions sur les territoires entre États et usagers locaux et de l'enchevêtrement, la superposition, voire parfois la contradiction entre des titres fonciers générés par l'État et les règles coutumières de gestion des ressources et des espaces. La situation est d'autant plus tendue que, dorénavant, de nombreux États «imposent» ou suggèrent fortement la 
cartographie participative comme outil de dialogue social, soit par une pression forte des médiateurs du territoire que sont les ONG, soit par une injonction légale.

8 L'article d'Hortensia Caballero traite spécifiquement de la dimension politique de la cartographie participative. L'exemple choisi, celui du Venezuela qui a reconnu des droits spécifiques aux populations autochtones dans sa Constitution de 1999, permet de mesurer l'ampleur des enjeux politiques autour de la carte. Pour des populations restées longtemps en marge de la société (socialement, économiquement et géographiquement), il s'agit d'obtenir des titres fonciers sur leurs terres mais en intégrant les marques de la " Nation », notamment ses frontières, dans les représentations du territoire.

\section{Le processus de cartographie est aussi intéressant à étudier, sinon plus, que la carte}

9 Plus qu'au seul produit fini nommé "carte", on s'intéresse d'abord aux objectifs revendiqués et mobilisés pour la réaliser et, d'une manière générale, aux processus qui aboutissent (ou non) à une carte consensuelle considérée comme un produit fini, publiable. Ce qu'il convient d'observer, c'est l'exercice de cartographie réalisé en collaboration et ce d'autant plus qu'il met en jeu des acteurs du territoire nombreux, aux objectifs et corpus de connaissances variés, et pas toujours habitués à se rencontrer. Etudier la cartographie c'est étudier plus largement les acteurs du territoire et leurs relations. Il est donc essentiel de porter une attention particulière au contexte géopolitique, aux acteurs mobilisés et aux étapes, y compris techniques, de la réalisation.

10 Ainsi, les 'leçons cartographiques' tirées de l'expérience menée par les Jodï et Eñepa (article de Stanford Zent et al.) montrent l'importance des alliances entre acteurs externes et internes pour tout projet de cartographie, qui permettent aux différents participants de reconnaitre qu'il existe d'autres manières de .percevoir et de pratiquer l'espace et le temps. En ce sens la cartographie est un outil complémentaire privilégié pour les études d'ethnoécologie menées sur le long terme ; la hiérarchisation, la description et finalement la spatialisation des savoirs locaux sur le milieu demandent à être travaillées en collaboration.

\section{Une carte d'identités multiples}

11 La cartographie participative, en s'affranchissant de la sémiologie du cartographe permet de représenter au-delà des simples éléments administratifs, de topographiques ou paysagers d'autres dimensions du territoire tout aussi importantes : symboliques, sacrées, toponymiques... Mais cartographier son lieu de vie, c'est aussi - ou d'abord - «pour les autres ", pour présenter une image de son territoire au reste du monde, une traduction acceptable de la façon dont chaque collectif pense ses liens aux lieux, ou l'organisation de son lieu de vie. Les produits finis, ces cartes, sont donc aussi des cartes d'identité : la projection d'une certaine perception de l'espace synthétisée et mise en scène pour l'extérieur. Il y a donc un biais intrinsèque à un exercice de cartographie, qui ne peut être la simple projection d'un espace perçu sur papier. À ce titre, ce qui est caché est au moins aussi important que ce qui est montré et une analyse des processus ne peut faire l'économie d'une connaissance anthropologique approfondie des sociétés étudiées. Il est sur ce point intéressant de noter que la carte participative est souvent mobilisée et 
appropriée par les communautés locales dans des situations d'insécurité foncière ou de mise en cause identitaire. C'est de fait le cas pour tous les exemples de cette première partie du dossier, quoique dans des termes et à des degrés divers.

Néanmoins puisqu'il s'agit d'une carte collective et non individuelle la question se pose de savoir de quelle « identité » parle-t-on, de sa représentativité par rapport au groupe, des règles sociales et des négociations qui régissent sa production et de la réalité de son caractère consensuel actuel. Ces discussions traversent de façon plus aigue les cartographies réalisées en contexte 'pluriethnique', ici au Venezuela et au Brésil, lorsqu'un même territoire est partagé par des populations reconnues culturellement différentes mais qui partagent de fait les mêmes références historiques et politiques.

Ailleurs, la carte peut être un élément susceptible de compter un jour pour asseoir une légitimité territoriale. Cartographier l'histoire, avec les déplacements d'un groupe donné, participe ainsi de processus de patrimonialisation et de récupérations territoriales comme le montre l'article de Marie Fleury et al. : en restituant une partie des résultats (les toponymes notamment) d'un travail de cartographie participative mené comme un voyage vers les origines, les auteurs proposent une relecture de l'histoire de la région en faisant dialoguer la tradition orale Wayana, l'histoire officielle écrite et les témoignages d'anciens voyageurs.

\section{Le rôle du chercheur}

Il nous faut encore souligner que les articles présentés dans ce dossier ont été écrits pour certains comme co-auteurs - par des chercheurs impliqués dans les processus de réalisation des cartes. Quels sont les différents rôles joués par les chercheurs dans ces processus: catalyseur, courtier, technicien cartographe, secrétaire, déclencheur ou simple observateur? Quels sont les apports de ces outils de science participative pour un chercheur en sciences sociales? Y-a-t-il une spécificité des processus de cartographie participative lorsque des chercheurs sont engagés dans le processus? Les différentes positions et postures des chercheurs dans les processus n'ont pas les mêmes implications et ouvrent des perspectives de recherche intéressantes et à haute réflexivité sur le degré d'engagement des chercheurs vis-à-vis de leur « objets » d'étude.

Dans tous les exemples présentés, la vitesse du processus (prônée comme qualité dans la plupart des méthodologies de cartographie participative mis en œuvre par les ONGs, puisque réalisée parfois en situation d'urgence, souvent à court-terme parfois pour prise de décision...) est ici dénoncée. Les chercheurs mettent au contraire l'accent sur une connaissance dans la durée des populations concernées, une confiance voire amitié construite sur un long-terme, comme un élément indispensable de la réussite du processus.

\section{Les limitations des outils cartographiques}

16 Les différentes contributions s'interrogent aussi sur les limitations, nombreuses, des outils. Les cartes sur support papier, qui font un choix de représentation ou qui figent une situation sont en fait mal adaptées à représenter les dynamiques des territoires, surtout lorsqu'ils sont partagées pour de multiples usages par de nombreux utilisateurs comme les forêts, les deltas, les espaces maritimes. Les outils de SIG ou de modélisation peuvent 
techniquement suppléer la carte papier en permettant la gestion de plusieurs couches d'information complexe, voire la modélisation des interactions. Cependant ces outils peuvent également être générateurs de barrières techniques et/ou cognitives qui peuvent parfois freiner l'appropriation du produit. Ainsi par exemple, l'article de Marie-Christine Cormier-Salem et al. montre bien les difficultés de développer un dispositif SIG pour la gouvernance du delta du fleuve Sénégal. La plateforme SIRENA a pour objectif de faciliter la représentation d'un espace à usages multiples, soumis à une forte variabilité spatiale et temporelle, en harmonisant les données spatiales. Cependant, dans un contexte de forte compétition entre institutions quant à la gouvernance de l'Aire Protégée, la méfiance initiale des gestionnaires quant à l'outil, ne se dissipe que par les rencontres entre acteurs lors de cycles de formation et la mise en pratique, dans la durée, de l'outil.

\section{Le devenir de la carte}

S'agissant d'une image figée d'un territoire, le regard porté sur la carte par ses propres producteurs peut lui-même être amené à évoluer. Il est donc important de non seulement étudier le processus de production d'une carte mais aussi son degré d'appropriation, ses utilisations lors de sa mise en circulation, ses réinterprétations et détournements possibles ou sa disparition dans l'oubli. Dans son article présentant les expériences successives de cartographie participative des "Peuples du Oiapoque ", Roselis Mazurek insiste sur l'importance du contexte des projets: on n'élabore pas les mêmes cartes et selon les mêmes processus quand il s'agit de faire reconnaitre des droits territoriaux à la société nationale, que quand il s'agit, plus tard, de discuter entre soi des modalités de gestion partagée d'un territoire acquis. Le degré d'appropriation du document et la profondeur de sa diffusion dans la société n'est pas non plus le même. Il apparaît également crucial de s'interroger plus systématiquement sur les méthodologies employées, notamment sur la réelle participation des habitants à chacune des étapes du processus de cartographie.

Nous espérons que cette première partie du dossier saura convaincre les lecteurs du caractère passionnant de l'étude des processus de cartographie participative aux Suds.

\section{NOTES}

1. Dans une deuxième partie du dossier "Cartographie Participative" qui sera publié en 2017, d'autres expériences menées par des chercheurs du laboratoire PALOC (IRD/MNHN), leurs collègues associés et des populations locales (Brésil, Guyane, Timor, Marquises, Afrique de l'Est) seront présentées. 


\section{AUTEURS}

\section{PASCALE DE ROBERT}

Anthropologue, Chargée de Recherche à l'IRD

UMR PALOC « Patrimoines Locaux et Gouvernance », MNHN - Département Hommes, Natures, Sociétés, CP 02657 rue Cuvier, 75231 Paris Cedex 05

\section{STÉPHANIE DUVAIL}

Géographe, Chargée de Recherche à l'IRD

UMR PALOC « Patrimoines Locaux et Gouvernance », MNHN - Département Hommes, Natures, Sociétés, CP 02657 rue Cuvier, 75231 Paris Cedex 05 